\title{
Interventional Radiologist
}

National Cancer Institute

\section{Source}

National Cancer Institute. Interventional Radiologist. NCI Thesaurus. Code C147951.

A radiologist that specializes in the use of minimally invasive image-guided procedures for the diagnosis and treatment of disease. 